\title{
Integrated Platform for Autonomic Computing
}

\author{
Christoforos Panayiotou \\ University of Cyprus \\ Nicosia, Cyprus \\ panchris@cs.ucy.ac.cy \\ Vassileios Tsetsos \\ National \& Kapodistrian University of Athens \\ Athens, Greece \\ b.tsetsos@di.uoa.gr \\ Stathes Hadjiefthymiades \\ National \& Kapodistrian University of Athens \\ Athens, Greece \\ shadj@di.uoa.gr
}

\author{
Eleftherios Fytros \\ Siemens A. E. Electrotechnical Projects and Products \\ Athens, Greece \\ Eleftherios.Fytros@siemens.com \\ George Samaras \\ University of Cyprus \\ Nicosia, Cyprus \\ cssamara@cs.ucy.ac.cy \\ Damien Piquet \\ Centre Suisse d'Electronique et de Microtechnique \\ Neuchatel, Switzerland \\ damien.piguet@csem.ch
}

\begin{abstract}
IPAC (Integrated Platform for Autonomic Computing) aims at delivering a middleware and service creation environment for developing embedded, intelligent, collaborative, context-aware services in mobile nodes. IPAC relies on short range communications for the ad hoc realization of dialogs among collaborating nodes. Advanced sensing components leverage the context-awareness attributes of IPAC, thus rendering it capable of delivering highly innovative applications for mobile and pervasive computing. IPAC networking capabilities are based on rumour spreading techniques, a stateless and resilient approach, and information dissemination among embedded nodes. Spreading of information is subject to certain rules (e.g., space, time, price). IPAC nodes may receive, store, assesses and possibly relay the incoming content to other nodes. The same distribution channel is followed for the dissemination of new applications and application components that "join the IPAC world".
\end{abstract}

Index Items: component; mobile middleware; autonomic applications; application creation environments; short range communications

\section{INTRODUCTION}

The proliferation of devices supporting ad-hoc networking (e.g., through the IEEE 802.11 protocol), like mobile phones, Personal Digital Assistants (PDAs), digital cameras, and portable music players (MP3 players), has resulted in a variety of popular applications and services. The use of ad-hoc networking for the communication between such "smart" devices has certain advantages compared to an infrastructurebased communication approach: 1) no global coordination is needed, 2) cost is reduced (no infrastructure is needed), and 3) coverage area of the network can be extended through multihop communication. Note, however, that the lack of

\footnotetext{
* This work was supported in part by the European Commission through the FP7 ICT Programme in the scope of the project IPAC (Integrated Platform for Autonomic Computing), contract FP7-ICT-224395.
}

infrastructure brings some challenges to the development of protocols, and services.

The smart devices need to cooperate with one another on a local basis so as to achieve a global goal dictated by the task that they serve. This includes the development of distributed communication protocols, as well as the development of applications and services in a way that takes into account the distributed nature of the discussed network. To cope with such a situation, network nodes need to take decisions on their own based only on information provided by the neighbouring environment, i.e., nodes must be autonomic.

In this proposal, we describe an Integrated Platform for Autonomic Communication (IPAC) [1]. IPAC aims at the delivery of a service creation and runtime (service provision) environment for autonomic communications. IPAC tries to address several challenges of autonomic computing, such as reliable and efficient algorithms for information dissemination in autonomic environments, developer-friendly application creation, automatic discovery of deployed sensors and knowledge-based node reconfiguration.

The main contributions of IPAC can be summarized as follows:

- A developer-friendly graphical user interface (GUI) for building and debugging IPAC applications. This GUI also comes with a custom application description language and a respective workflow language that enable developers to write applications in an abstract way.

- Short range communication technologies that support a novel probabilistic information dissemination model. This model is based on the concepts of [2] and is appropriate for environments with nomadic nodes with limited energy resources.

- Knowledge-based reconfiguration for embedded systems. IPAC is one of the first attempts to support 
mobile reasoning and relevant mechanisms for providing service intelligence. Similar work in this area can be found in [3][4].

- Adoption of the IEEE 1451 [5] standard for implementing plug and play sensors. IEEE 1451 is an evolving standard that promises a new era of sensorenabled applications, through easy integration of diverse sensor technologies.

- Collaborative context-awareness. IPAC nodes can use contextual information for adapting the application execution even if they do not have attached sensors. Specifically, they can "harvest" sensor data and contextual events from their local neighbourhood through a publish/subscribe mechanism.

\section{MAIN TECHNICAL OBJECTIVES}

The lightweight and flexible IPAC middleware provides all services required for the deployment and execution of diverse applications in a collaborative nomadic environment. These services are supported by novel knowledge and ontology engineering techniques, dealing with interoperability, integration, and re-configuration / adaptation problems encountered in contemporary embedded platforms. Figure 1 presents an overview of the IPAC middleware. Being collaborative, IPAC relies on short-range communications (e.g., ZigBee, DSRC, and Bluetooth) for the ad hoc realization of dialogs between nodes. Being context-aware, IPAC relies on advanced sensing components thus, delivering highly innovative application architecture.

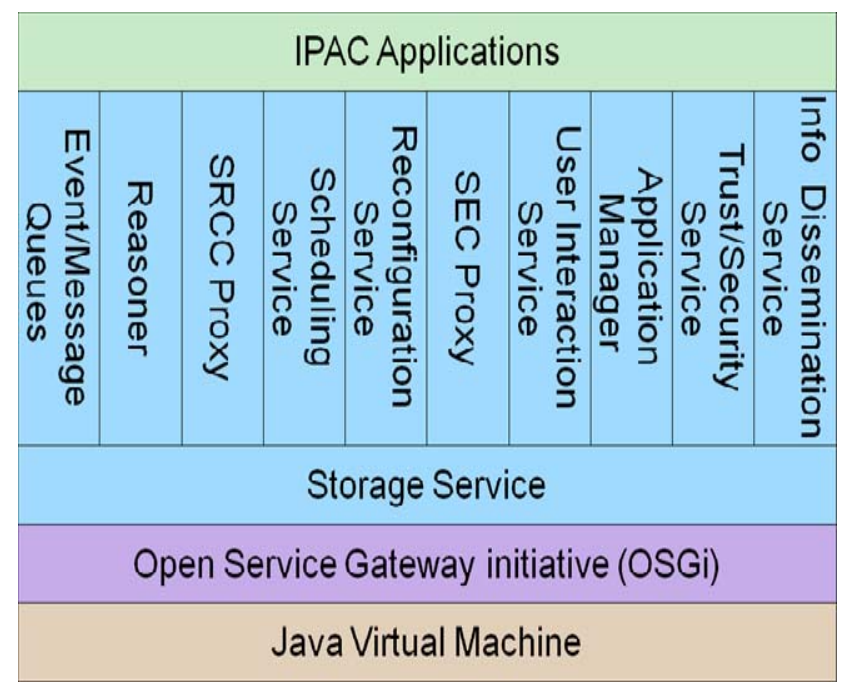

Figure 1. IPAC Middleware

IPAC is based on sophisticated information dissemination algorithms [6]. Specifically, it relies on rumour spreading techniques. Rumour spreading involves the propagation of information within a certain network. Information is ducted only to immediate neighbours that are interested in specific content (rumour). Therefore, IPAC incorporates recent research advances in the area of bio-inspired computing systems.

Mobile nodes in IPAC are specified and developed to be non-selfish with respect to information dissemination (a mobile incarnation of a peer-to-peer system). Specifically, the IPAC nodes operate in a collaborative fashion in order to diffuse contextual information and broader knowledge in their environment. A node propagates an information message received by another node across the network. In case that such message appears to be usable for the node, it can process it. An information message that is of no interest to an IPAC node has to be forwarded across the network for further processing. The same path is followed for the dissemination of new applications or application components after their development thus contributing to the deployment and use of new embedded applications. IPAC integrates techniques and algorithms for energy-efficient, autonomic node behaviour, advanced context awareness, embedded service/application modelling and efficient information dissemination.

\section{TECHNICAL APPROACH}

IPAC pursues the development of a middleware platform for embedded devices with specific characteristics. IPAC also aims at the delivery of an application creation and runtime environment for autonomic computing. Autonomic nodes enter in the proximity of other nodes and relay relevant information in the form of "rumour spreading". Target applications include traffic management, in-building guidance, industrial environment control, as well as crisis management. For instance, vehicles equipped with an IPAC device may forward information to one another regarding the road conditions (e.g., congestion, accidents, etc.) so as to improve circulation, and avoid potential accidents. Another potential application domain of IPAC is road advertising, or advertising in large commercial centres. Individuals in a shopping centre obtain useful and timely information (e.g., where a specific product may be found, discounts, special offers, etc) through the autonomous information exchanging of their IPAC devices.

The platform is supplemented by an application creation environment. Within the scope of IPAC, the application creation environment refers to all tools, APIs and libraries, which assist the development of new applications for IPAC nodes. It is responsible for providing a sophisticated interface to developers in order to be able to define applications as well as their properties. This is to be done in an efficient, userfriendly manner that also allows for reusability of design and code. It is a software module runnable on a standard computer and coexists and cooperates with well-known third party software. It will provide a visual and a textual interface (see Figure $2 \& 3$ ) to developers and tools for testing and emulating every application before their deployment in IPAC nodes.

Applications may be preinstalled on the node or deployed on demand by the user. Nodes may be considered as sources of information stemming from various sensors mounted on them or from human user input. Such information may be disseminated from one node to another in the network, thus, catering for a distributed, autonomic information propagation 
platform. In order to have meaningful and controlled information dissemination, the spreading of information is governed by space-time validity rules (directives). For instance, a message concerning congestion at a crossroad would be valid for less than one hour and within a radius of some kilometres.

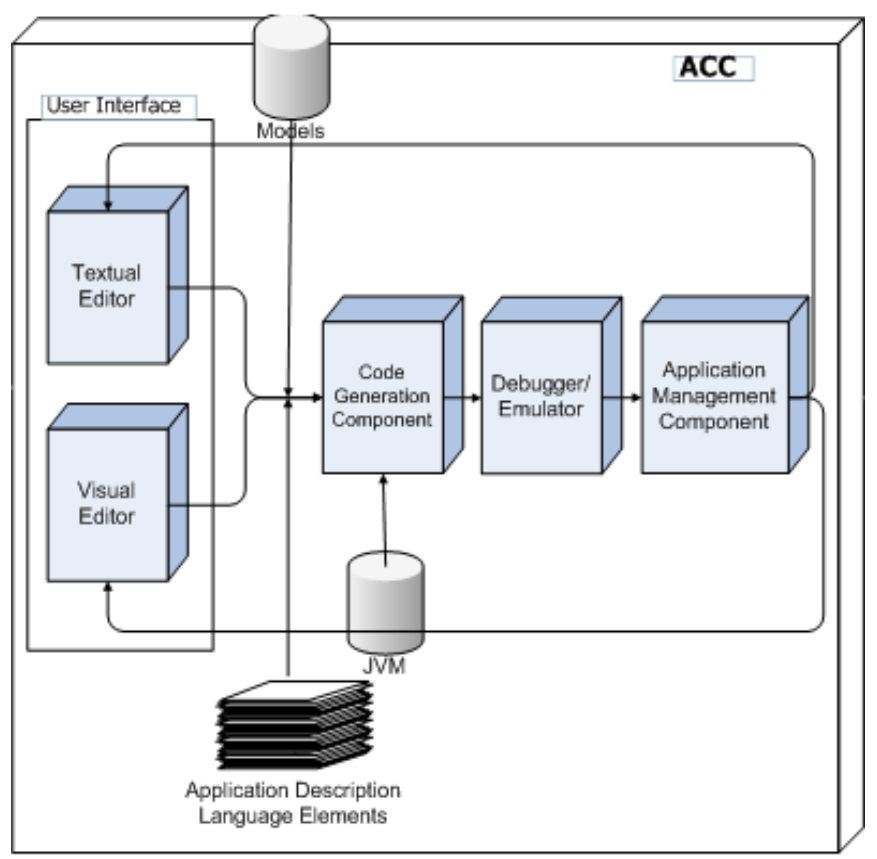

Figure 2. Application Creation Component architecture

The IPAC uses the Short Range Communication (SRC) technology for achieving communication between autonomic entities, thus, enhancing bandwidth and reducing latency in the communication link. Contrary to other wireless technologies (e.g., GSM) SRC operates in short distances, thus, enabling the formation of relatively small and isolated communication zones. In addition, IPAC explores the possibility of applying wireless sensor network technologies for implementing shortrange communications. An indicative example of such a technology is the 802.15.4 protocol (ZigBee) targeting to devices with low battery consumption constraints that need to continuously transmit information.

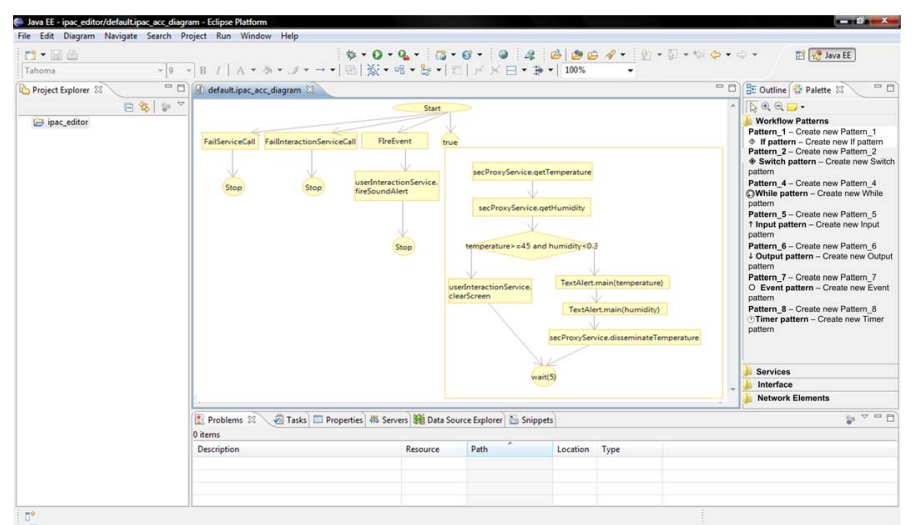

Figure 3. Application Creation Component implemented as an Eclipse plugin

\section{KEY ISSUES}

IPAC develops embedded middleware technology for the realization of innovative context-aware services by autonomous nodes. Supported by ad-hoc network infrastructures, IPAC proceeds to the study of situation- and context-aware services deployed by numerous and mostly dynamic network groups and communities. Context-awareness allows autonomic nodes to sense and adapt to their environment, not only at the network level but also at the application plane. This adaptive behaviour will be supported by knowledge-based methods and technologies, in a highly innovative manner.

Therefore, the key contribution of IPAC is a novel embedded middleware and service provision platform that brings considerable intelligence to the device. None of the existing research projects or products, in the academic or industrial context, has implemented all the self-CHOP characteristics (self-configuration, self-healing, self-optimizing and self-protecting), and additionally offer self-awareness and context-awareness, which are of major importance in many environments. IPAC addresses all the self-CHOP requirements and thus provides a solid, efficient and future-proof platform.

\section{EXPECTED IMPACT}

The expected impact of IPAC spreads beyond the strict limits of the embedded devices sector. The applications supported have a profound impact on multiple human activities. Some example applications are: road safety (through embedded sensor networks both in vehicles and public roads), manufacturing (both through quality control checks and improved feedback to the actual manufacturing robots), energy conservation (through power-efficiency mechanisms for energy consumption), monitoring of core and metropolitan power, targeted applications (advertising) and crisis management (through the creation of ad hoc communication infrastructure).

\section{REFERENCES}

[1] IPAC project: http://ipac.di.uoa.gr

[2] D. Demers, D. Greene, C. Hauser, W. Irish, J. Larson, S. Shenker, H. Sturgis, D. Swinehart, D. Terry, "Epidemic Algorithms for Replicated Database Maintenance", Proc. ACM Principles of Distributed Computing, 1-12, 1987.

[3] O. Noppens, M. Luther, T. Liebig, M. Wagner, M. Paolucci. Ontologysupported Preference Handling for Mobile Music Selection, Conference Paper, ECAI'06 Workshop on Advances in Preference Handling (AdvPref'06), August 2006.

[4] Gu T., Kwok Z., Koh KK. and Pung HK. A Mobile Framework Supporting Ontology Processing and Reasoning. In proc. 2nd Workshop on Requirements and Solutions for Pervasive Software Infrastructure (RSPS) in conjunction with the 9th International Conference on Ubiquitous Computing (Ubicomp '07), September, Austria, 2007.

[5] Janusz Bryzek, Introduction to IEEE-P1451, the emerging hardwareindependent communication standard for smart transducers, Sensors and Actuators A: Physical, Volume 62, Issues 1-3, Proceedings of Eurosensors X, July 1997, Pages 711-723, ISSN 0924-4247, DOI: 10.1016/S0924-4247(97)01536-7.

[6] M.J. Franklin, S.B. Zdonik, "Dissemination-based information systems", Data Engineering Bulletin, 19(3), 20-30, 1996. 